\title{
A PDE Approach to the Derivation of the Brayton-Moser Form for Power-Shaping Control
}

\author{
Audrey Favache, Denis Dochain and Joseph J. Winkin
}

\begin{abstract}
Power-shaping control is an extension of energybalancing passivity-based control based on a particular form of the dynamics, the Brayton-Moser form. One of the main difficulties in this approach is to write the dynamics in the suitable form since it requires the solution of a partial differential equation (PDE) system with an additional sign constraint. Here a general methodology is described for solving this partial differential equation system. The set of all solutions to the PDE system is given as the solution of a linear equation system. A necessary condition is given so that a solution of the linear system which meets the sign condition exists. This methodology is illustrated on a chemical reactor example, where the physical knowledge of the system is used to find a suitable solution.
\end{abstract}

\section{INTRODUCTION}

Power-shaping control [17] has been developed in the past years as an extension of energy-balancing passivity-based control [12][18]. In energy-balancing passivity based control, the controller reshapes the energy function of the system so that it has a minimum at the desired equilibrium point. The controller provides the system with a finite amount of energy so as to drive the system to the desired state. This concept has been applied to electro-mechanical systems [15][19] and also to thermodynamic systems where the storage function is the entropy instead of the energy [1][20]. Nevertheless energy-balancing passivity-based control cannot be applied to some systems (namely systems with pervasive dissipation). To overcome this difficulty the concept of power-shaping control has been introduced, primary for the stabilization of nonlinear RLC circuits [17]. Contrary to energy-balancing passivity-based control, the storage function used for the control is related to the power and not to the energy. Power-shaping control has subsequently been applied to the control of (electro)mechanical systems [9], and also chemical reaction systems [5][6][7].

The power-shaping control methodology is based on a particular formulation of the system dynamics, namely the Brayton-Moser form. This is, as the authors state in [9], one of the major difficulties of applying this control methodology since it requires the solution of a partial differential equation (PDE) system subjected to a sign constraint. Up to now, no general solution has been found to this problem. In this framework we propose a general methodology that allows to build the Brayton-Moser form of the dynamics. First we

A. Favache is with IMMC, Université catholique de Louvain, 2 place Sainte Barbe, B-1348 Louvain-la-Neuve, Belgium, e-mail : audrey.favache@uclouvain.be; D. Dochain is with CESAME, Université Catholique de Louvain, 4-6 avenue G. Lemaîitre, B-1348 Louvain-la-Neuve, Belgium, e-mail : denis.dochain@uclouvain.be; J. J. Winkin is with NaXys and Department of Mathematics, University of Namur (FUNDP), 8, Rempart de la Vierge, B-5000 Namur, Belgium, e-mail : joseph.winkin@ @undp.ac.be shall briefly recall the context of the Brayton-Moser PDE, namely power-shaping control (Section II). The main part of this work shall be presented in Section III where the solution methodology is explained. Finally in Section IV this methodology is illustrated by the example of a continuous stirred tank reactor (CSTR).

\section{Power-Shaping CONTROL AND BRAYTON-Moser FORM}

\section{A. Power-shaping control}

Let us consider a dynamical system of dimension $N$ with $m$ inputs. The state of the system is given by the vector $x \in \mathbb{R}^{N}$ and the input is given by the vector $u_{c} \in \mathbb{R}^{m}$. The power-shaping control theory is based on the Brayton-Moser formulation of the system dynamics [2]. In this formulation the system dynamics are of the following form :

$$
\boldsymbol{Q}(x) \frac{d x}{d t}=\nabla \mathscr{P}(x)+G(x) u_{c}
$$

where $Q: D \rightarrow \mathbb{R}^{N} \times \mathbb{R}^{N}$ is a non-singular square matrix function, $\mathscr{P}: D \rightarrow \mathbb{R}$ is a scalar function of class $\mathcal{C}^{2}$, $G: D \rightarrow \mathbb{R}^{N} \times \mathbb{R}^{m}$ is a matrix function and $D$ is an open subset of $\mathbb{R}^{N}$. Additionally the symmetric part of the matrix $\boldsymbol{Q}(x)$ is negative semi-definite, i.e.:

$$
\boldsymbol{Q}(x)+\boldsymbol{Q}^{t}(x) \preceq 0 .
$$

The function $\mathscr{P}(x)$ is called the potential function. In electrical and mechanical systems, the potential function has the units of power and is related to the dissipated power in the system. In electrical (mechanical, resp.) systems it is related to the so-called content and co-content of the resistances [17] (Rayleigh dissipation function, resp. [13][14]).

In the power-shaping approach the controller is designed such as to re-shape the potential function so that it has a minimum at the desired closed-loop equilibrium. The input $u_{c}(x)$ is chosen such that in closed loop the system dynamics are given by the following relation :

$$
\boldsymbol{Q}(x) \frac{d x}{d t}=\nabla \mathscr{P}_{d}(x)
$$

where $\mathscr{P}_{d}: D \rightarrow \mathbb{R}$ is the re-shaped potential function. If $D$ fulfills some suitable assumptions, the function $\mathscr{P}_{d}(x)$ can be used as a Lyapunov function for the closed-loop system, thanks to the sign constraint (2). 


\section{B. Brayton-Moser form}

Originally the Brayton-Moser form of the dynamics has been developed for electric circuits [2]. Later, it has been shown that mechanical systems can be written in this form too [13][14]. However power-shaping control can be applied to any system that can be put in the form (1). In [9] conditions for writing a dynamical system in the form (1) are given.

Let us assume that the system dynamics are given by the following relation :

$$
\frac{d x}{d t}=f(x)+g(x) u_{c}
$$

where $f: D \rightarrow \mathbb{R}^{N}$ is a vector function, $g: D \rightarrow \mathbb{R}^{N} \times \mathbb{R}^{m}$ is a matrix function and $D$ is an open simply connected subset of $\mathbb{R}^{N}$. The system (4) can be written in the form (1) if there exists a non-singular matrix function $\boldsymbol{Q}(x)$ fulfilling (2) and that solves the following PDE system ${ }^{1}[9]$ :

$$
\nabla(\boldsymbol{Q}(x) f(x))=\nabla^{t}(\boldsymbol{Q}(x) f(x)),
$$

where $M^{t}$ denotes the transpose of a matrix $M . \mathscr{P}(x)$ is then a solution of the following PDE system:

$$
\nabla \mathscr{P}(x)=\boldsymbol{Q}(x) f(x)
$$

and the function $G(x)$ is given by $G(x)=\boldsymbol{Q}(x) g(x)$. Condition (5) means that the Hessian matrix of the potential function $\mathscr{P}(x)$ is symmetric. By Schwarz-Young theorem, this condition is necessary for $\mathscr{P}(x)$ to be twice differentiable.

\section{SOLUTION METHODOLOGY}

According to the previous section, the power-shaping control approach can be applied to any dynamical system provided that a solution to the Brayton-Moser PDE (5) satisfying the sign constraint (2) can be found. This condition is the main limitation for applying the power-shaping control approach. In this section a general methodology is described for finding a solution to this problem.

\section{A. Solution to the PDE system}

Let us consider a dynamical system whose dynamics are given by (4). The PDE system to solve is given by (5). In the following proposition, the existence of a solution to (5) is established by a constructive proof.

Proposition 1. If $f(x)$ is twice continuously differentiable, then a solution $\boldsymbol{Q}(x)$ to (5) always exists such that (6) holds for a suitable a priori choice of potential function $\mathscr{P}(x)$.

Proof: Let us denote by $q_{i j}$ the element in position $(i, j)$ of the unknown matrix $\boldsymbol{Q}(x)=\left(q_{i j}(x)\right)$. Let us also define

\footnotetext{
${ }^{1}$ This condition is equivalent to the existence of the potential function $\mathscr{P}(x)$.
}

the vector $z(x)$ whose components are given as follows $(1 \leq$ $i, j \leq N)$ :

$$
z(x)=\left(\begin{array}{c}
z_{1}(x) \\
z_{2}(x) \\
\vdots \\
z_{N}(x) \\
z_{N+1}(x) \\
\vdots \\
z_{(i-1) N+j}(x) \\
\vdots \\
z_{N^{2}}(x)
\end{array}\right)=\left(\begin{array}{c}
q_{11}(x) \\
q_{12}(x) \\
\vdots \\
q_{1 N}(x) \\
q_{21}(x) \\
\vdots \\
q_{i j}(x) \\
\vdots \\
q_{N N}(x)
\end{array}\right)
$$

Consider the set $E$ of the equilibria of the unforced system :

$$
E=\{\bar{x} \in D \mid f(\bar{x})=0\}
$$

Let us choose a twice continuously differentiable scalar function $\mathscr{P}(x)$ defined on $D$ and such that

$$
\nabla \mathscr{P}(\bar{x})=0 \quad \text { for all } \bar{x} \in E
$$

It is always possible to find a function $\mathscr{P}(x)$ that fulfills this condition, for example $\mathscr{P}(x)=\|f(x)\|_{2}^{2}=\sum_{k=1}^{N}\left(f_{k}(x)\right)^{2}$ (which is of class $\mathcal{C}^{2}$, since so is $f(x)$ ).

Now consider the following linear algebraic system :

$$
A(x) z(x)=\left(\begin{array}{c}
\frac{\partial \mathscr{P}}{\partial x_{1}} \\
\vdots \\
\frac{\partial \mathscr{P}}{\partial x_{N}}
\end{array}\right)
$$

with

$$
A(x)=\left(\begin{array}{cccc}
f_{1}(x) f_{2}(x) \cdots f_{N}(x) & 0 & \cdots & 0 \\
0 & f^{t}(x) & \cdots & 0 \\
\vdots & \vdots & \ddots & \vdots \\
0 & 0 & \cdots & f^{t}(x)
\end{array}\right)
$$

This system matrix rank is equal to $N$ if $x \notin E$ and to 0 if $x \in E$. Hence $\operatorname{Im}(A(x))=\mathbb{R}^{N}$ if $x \notin E$, and $\operatorname{Im}(A(x))=$ $\{0\}$ if $x \in E$. For any fixed $x$, (9) has an infinite number of solutions if and only if $\nabla \mathscr{P}(x) \in \operatorname{Im}(A(x))$. In the other cases (9) has no solution. The way $\nabla \mathscr{P}(x)$ has been chosen (i.e. such that (8) holds) obviously ensures that $\nabla \mathscr{P}(x) \in$ $\operatorname{Im}(A(x))$. Consequently there exists an infinite number of solutions to (9).

On the other hand the Brayton-Moser PDE system (5) can be written as follows :

$$
\sum_{k=1}^{N} \frac{\partial\left(q_{i k} f_{k}\right)}{\partial x_{j}}=\sum_{k=1}^{N} \frac{\partial\left(q_{j k} f_{k}\right)}{\partial x_{i}}, \quad 1 \leq i, j \leq N .
$$

The solutions of (9) fulfill the following relation :

$$
\sum_{k=1}^{N} f_{k} z_{(i-1) N+k}=\sum_{k=1}^{N} f_{k} q_{i k}=\frac{\partial \mathscr{P}}{\partial x_{i}}, \quad 1 \leq i \leq N,
$$


i.e. (6) holds. Thanks to the fact that $\mathscr{P}(x)$ has been chosen of class $\mathcal{C}^{2}$, it follows from (10) and (11):

$$
\sum_{k=1}^{N} \frac{\partial\left(q_{i k} f_{k}\right)}{\partial x_{j}}=\frac{\partial}{\partial x_{j}}\left(\frac{\partial \mathscr{P}}{\partial x_{i}}\right)=\frac{\partial^{2} \mathscr{P}}{\partial x_{i} \partial x_{j}}
$$

Hence the solutions of (9) fulfill (10) and therefore yield a solution of the Brayton-Moser PDE system (5). Since there exist an infinite number of solutions to (9), there exists also an infinite number of solutions to (5).

The proof of the proposition above gives a way to parametrize all the solutions of (5). Indeed once a function $\mathscr{P}(x)$ has been chosen, a solution of the undetermined linear system (9) is a solution of (5). Hence the set of solutions of (5) can be written as the sum of the general solution of the homogeneous system $A(x) z(x)=0$ (i.e. $z(x) \in$ ker $A(x)$ ) and a particular solution of the non-homogeneous system (9). Without loss of generality, let us assume that $f_{1}(x) \neq 0$ (for $x \notin E$ ). Then a basis for $\operatorname{ker} A(x)$ is given by the vectors $e^{(j-1) N+i}$ with $j=1, \ldots, N$ and $i=2, \ldots, N$ :

$$
\begin{aligned}
& e^{(j-1) N+i}(x)= \\
& {[\underbrace{0 \cdots 0}_{(j-1) N \text { times }}-\frac{f_{i}(x)}{f_{1}(x)} \underbrace{0 \cdots 0}_{(i-2) \text { times }} 1 \underbrace{0 \cdots 0}_{(N-i+N(N-j)) \text { times }}]^{t}}
\end{aligned}
$$

and a particular solution of (9) is given by:

$$
\begin{aligned}
z(x)= & {[\frac{\partial \mathscr{P}}{\partial x_{1}} \frac{1}{f_{1}(x)} \underbrace{0 \cdots 0}_{(N-1) \mathrm{times}} \frac{\partial \mathscr{P}}{\partial x_{i}} \frac{1}{f_{1}(x)} \underbrace{0 \cdots 0}_{(N-1) \text { times }}} \\
& \cdots \frac{\partial \mathscr{P}}{\partial x_{N}} \frac{1}{f_{1}(x)} \underbrace{0 \cdots 0}_{(N-1) \text { times }}]^{t}
\end{aligned}
$$

The next proposition shows that all the solutions of (5) are of the form suggested in the proof of Proposition 1.

Proposition 2. If $D$ is an open simply connected subset of $\mathbb{R}^{N}$, all the solutions $\boldsymbol{Q}(x)$ of (5) are such that there exists a function $\mathscr{P}(x)$ that fulfills (9).

Proof: Let us consider a solution $\boldsymbol{Q}(x)$ of (5). Let us define the vector $v$ as follows: $v_{i}=\sum_{j=1}^{N} q_{i j} f_{j}(x)$. Since $\boldsymbol{Q}(x)$ is a solution of (5), the following relations hold : $\frac{\partial v_{i}}{\partial x_{j}}=\frac{\partial v_{j}}{\partial x_{i}}, \quad 1 \leq i, j \leq N$. Let us define the following 1-form:

$$
w=\sum_{i=1}^{N} v_{i} \mathrm{~d} x_{i}
$$

Observe that the exterior derivative $\mathrm{d} w$ of $w$ is identically zero. Following Poincaré's theorem since $D$ is an open simply connected subset of $\mathbb{R}^{N}$ and $w$ a differential form which is closed (since $\mathrm{d} w=0$ on $D$ ), $w$ is an exact form ${ }^{2}$.

\footnotetext{
${ }^{2}$ see [16], Lemma 7.9, page 183.
}

As a consequence there exists a 0 -form $\theta=\mathscr{P}(x)$ such that the following relation is fulfilled:

$$
w=\mathrm{d} \theta=\sum_{i=1}^{N} \frac{\partial \mathscr{P}}{\partial x_{i}} \mathrm{~d} x_{i} .
$$

Equating (14) and (15) allows to write the following relation:

$$
v_{i}(x)=\frac{\partial \mathscr{P}}{\partial x_{i}}=\sum_{j=1}^{N} q_{i j}(x) f_{j}(x) \quad 1 \leq i \leq N,
$$

which is indeed the linear system (6) or equivalently (9). It is obvious from (16) that $\mathscr{P}(x)$ is such that $\nabla \mathscr{P}(\bar{x})=0$ for all $x \in E$ (with $E$ defined by (7)) since $f(\bar{x})=0$.

An additional requirement is that $\boldsymbol{Q}(x)$ is not singular. The following proposition gives a necessary condition on $\mathscr{P}(x)$. This result is a straightforward consequence of Proposition 2.

Proposition 3. Any solution $\boldsymbol{Q}(x)$ of (5) built using the methodology proposed in the proof of Proposition 1 is non-singular only if $\nabla \mathscr{P}(\bar{x})=0$ for all $\bar{x} \in E$ and $\nabla \mathscr{P}(x) \neq 0$ for all $x \in D, x \notin E$ (with $E$ defined by (7)).

\section{B. Sign constraint}

The methodology presented above allows to build a solution of (5). However, in order to be suitable for control design, the matrix $\boldsymbol{Q}(x)$ of the Brayton-Moser form must also fulfill the sign condition (2). A first approach could be to build an arbitrary solution to (5) as suggested above and then to use the methodology presented in [9] to build other solutions departing from the first one, and find one that fulfills the sign condition. However this can rapidly become tedious with increasing system size, all the more so since we do not even know if such a solution exists.

In the following proposition, whose proof is straightforward, a necessary condition on $\mathscr{P}(x)$ is given such that there exists a solution of the linear system (9) that fulfills the sign condition (2).

Proposition 4. The algebraic system (9) can have solutions which fulfill (2) only if $\mathscr{P}(x)$ is non-increasing along the trajectories of the unforced system (4).

Remark. As indicated above, any solution $\boldsymbol{Q}(x)$ of (6) (or equivalently of (9)) has the form $\boldsymbol{Q}(x)=\boldsymbol{Q}_{p}(x)+\boldsymbol{Q}_{h}(x)$, where $\boldsymbol{Q}_{p}(x)$ is a particular solution and $\boldsymbol{Q}_{h}(x)$ is any solution of the homogeneous equation $\boldsymbol{Q}_{h}(x) f(x)=0$. Observe that, for all $x \notin E, \Delta(x)=\operatorname{span}\{f(x)\} \subset \mathbb{R}^{N}$ is a one-dimensional nonsingular distribution, where $\Delta(x)$ is involutive. It follows by Frobenius theorem ${ }^{3}$ that $\Delta(x)$ is completely integrable, i.e. there exist scalar functions

${ }^{3}$ see [11] page 23 . 
$\lambda_{1}, \ldots, \lambda_{N-1}$ whose differentials span the annihilator of $\Delta(x)$. Hence $\boldsymbol{Q}_{h}(x)$ can be parametrized as follows:

$$
\boldsymbol{Q}_{h}(x)=\boldsymbol{Q}_{c} \cdot \nabla L(x)
$$

where

$$
\nabla L(x)=\left[\begin{array}{c}
\nabla \lambda_{1} \\
\vdots \\
\nabla \lambda_{N-1}
\end{array}\right]
$$

and $\boldsymbol{Q}_{c} \in \mathbb{R}^{N \times(N-1)}$ is a constant parameter matrix. Then the sign constraint (2) is equivalent to the linear matrix inequality : $\boldsymbol{Q}_{c} \nabla L+(\nabla L)^{t} \boldsymbol{Q}_{c}^{t}+\boldsymbol{Q}_{p}+\boldsymbol{Q}_{p}^{t} \leq 0$.

\section{ApplicAtion}

\section{A. Dynamical model of the CSTR}

Let us consider a liquid-phase continuous stirred tank reactor (CSTR) with constant volume $V$ containing two species $A$ and $B$ and in which a reversible reaction $A \rightleftharpoons \nu B$ takes place. The reactor is cooled/heated by a surrounding jacket. As it has been shown in [8] the dynamics of such a system are given by the following equations:

$$
\left\{\begin{aligned}
\frac{d n_{A}}{d t} & =\frac{F}{V}\left(C_{A}^{i n} V-n_{A}\right)-r\left(T, n_{A}, n_{B}\right) V \\
\frac{d n_{B}}{d t} & =\frac{F}{V}\left(C_{B}^{i n} V-n_{B}\right)+\nu r\left(T, n_{A}, n_{B}\right) V \\
\frac{d U}{d t} & =\frac{F}{V}\left(h^{i n} V-H\right)+\dot{Q}
\end{aligned}\right.
$$

with $n_{i}$ the quantity of species $i(i=A, B), U$ the internal energy of the mixture, $F$ the volumetric flow rate, $V$ is the liquid volume, $C_{i}^{i n}$ is the concentration of $i$ in the inlet flow, $r$ is the reaction rate, $h^{i n}$ is the volumetric enthalpy of the inlet flow, $T$ the temperature, $H$ the enthalpy of the mixture and $\dot{Q}$ the heat transferred into the reacting mixture. $H$ and $T$ are functions of $n_{A}, n_{B}$ and $U$ depending on the thermodynamic model of the liquid (e.g. an ideal liquid mixture):

$$
H=\hat{H}\left(n_{A}, n_{B}, U\right), \quad T=\hat{T}\left(n_{A}, n_{B}, U\right)
$$

The reaction rate function is assumed to be a twice continuously differentiable function such that :

$$
\begin{aligned}
& \text { - } r\left(\hat{T}\left(n_{A}, n_{B}, U\right), n_{A}, n_{B}\right) \leq 0 \text { if } n_{A}=0 \\
& \text { - } r\left(\hat{T}\left(n_{A}, n_{B}, U\right), n_{A}, n_{B}\right) \geq 0 \text { if } n_{B}=0
\end{aligned}
$$

The aim is to control the reactor temperature $T$ by acting on the heat transfer $\dot{Q}$. Using the notations of (4) we have :

$$
\begin{aligned}
f\left(n_{A}, n_{B}, U\right)= & \left(\begin{array}{c}
-\hat{r}\left(n_{A}, n_{B}, U\right) V \\
\nu \hat{r}\left(n_{A}, n_{B}, U\right) V \\
0
\end{array}\right) \\
g\left(n_{A}, n_{B}, U\right)= & \left(\begin{array}{cc}
C_{A}^{i n} V-n_{A} & 0 \\
C_{B}^{i n} V-n_{B} & 0 \\
V h^{i n}-\hat{H}\left(n_{A}, n_{B}, U\right) & 1
\end{array}\right)
\end{aligned}
$$

with $\hat{r}\left(n_{A}, n_{B}, U\right)=r\left(\hat{T}\left(n_{A}, n_{B}, U\right), n_{A}, n_{B}\right)$ and $u_{c}=$ $\left[\frac{F}{V} \dot{Q}\right]^{t}$. The sign conditions above on the reaction rate $r$ guarantee that the function $f:[0, \infty)^{3} \rightarrow \mathbb{R}^{3}$ is essentially nonnegative. Hence by [4] (Theorem 1), with $x=$ $\left(n_{A}, n_{B}, U\right)^{t}$, the nonnegative orthant $[0, \infty)^{3}$ is an invariant set with respect to the unforced system $\dot{x}=f(x)$.

\section{B. Solution of the Brayton-Moser PDE}

1) Potential function candidate: Following the methodology proposed in Section III the first step consists in choosing a suitable function $\mathscr{P}(x)$. According to Proposition 4 this function has to be decreasing along the system trajectories. One may believe that a priori the opposite of the entropy function could be a suitable potential function candidate. Indeed the unforced system is an isolated system and, according to the second principle of thermodynamics, in such systems the entropy is increasing. However the gradient of the entropy is not equal to zero at the equilibria of an isolated system. Originally in the powershaping approach the potential function has power units. By analogy, we shall consider here the entropy production as a potential function candidate, i.e. $\mathscr{P}(x)=\sigma_{S}$. Indeed for isolated systems, the entropy production is zero at the equilibrium and hence the entropy production has a local minimum. In [10] a general evolution criterion is given for all macroscopic systems submitted to time independent boundary conditions. For some thermodynamic systems, this criterion implies that the entropy production is decreasing along the trajectories.

For an isolated system the entropy production is equal to the entropy variation:

$\sigma_{S}=\frac{d S}{d t}=\left(\frac{\partial \hat{S}}{\partial x}\right)^{t} \frac{d x}{d t}=\left(\frac{\mu_{A}}{T}-\nu \frac{\mu_{B}}{T}\right) \hat{r}\left(n_{A}, n_{B}, U\right) V$

where $\hat{S}\left(n_{A}, n_{B}, U\right)$ is the entropy as a function (which is assumed to be of class $\mathcal{C}^{2}$ ) of $n_{A}, n_{B}, U$ and $\mu_{i}$ is the chemical potential of species $i$. Indeed from thermodynamic theory we have $\frac{\partial \hat{S}}{\partial x}=\left(-\frac{\mu_{A}}{T},-\frac{\mu_{B}}{T}, \frac{1}{T}\right)^{t}$ [3]. The following conditions will be assumed to hold throughout.

Assumption 1. The reaction kinetics are such that $\Lambda \hat{r}\left(n_{A}, n_{B}, U\right) \geq 0$ where $\Lambda=\frac{\mu_{A}}{T}-\nu \frac{\mu_{B}}{T}=$ $-(1-\nu 0) \frac{\partial \hat{S}}{\partial x}$ is the reaction affinity, and $\Lambda=0$ if and only if

$$
\hat{r}\left(n_{A}, n_{B}, U\right)=0 .
$$

Assumption 2. The reaction kinetics $r\left(T, n_{A}, n_{B}\right)$ are such that $\frac{d \sigma_{S}}{d t} \leq 0$ along the system trajectories with $\frac{d \sigma_{S}}{d t}=0$ only if $r\left(T, n_{A}, n_{B}\right)=0$.

Assumption 3. The limits

$$
\lim _{\hat{r} \rightarrow 0} \frac{\partial \sigma_{S}}{\partial n_{A}} \frac{1}{\hat{r} V} \text { and } \lim _{\hat{r} \rightarrow 0} \frac{\partial \sigma_{S}}{\partial n_{B}} \frac{1}{\hat{r} V}
$$

are defined and finite. 
Assumption 1 is necessary to ensure that the second principle of thermodynamics is fulfilled, namely that the entropy production is always non-negative. It means that the reaction always evolves in the direction of decreasing affinity.

It can be shown that assumption 2 is additionnaly required to ensure that the entropy production is decreasing along the system trajectories and can therefore be used for the function $\mathscr{P}(x)$.

The assumptions above can be satisfied for instance by the classical mass action balance kinetics with Arrhenius type dependence [21].

2) Computation of $\boldsymbol{Q}(x)$ : The linear system (9) reads as follows:

$$
\left\{\begin{array}{l}
{\left[\nu q_{12}\left(n_{A}, n_{B}, U\right)-q_{11}\left(n_{A}, n_{B}, U\right)\right] \hat{r} V=\frac{\partial \sigma_{S}}{\partial n_{A}}} \\
{\left[\nu q_{22}\left(n_{A}, n_{B}, U\right)-q_{21}\left(n_{A}, n_{B}, U\right)\right] \hat{r} V=\frac{\partial \sigma_{S}}{\partial n_{B}}} \\
{\left[\nu q_{32}\left(n_{A}, n_{B}, U\right)-q_{31}\left(n_{A}, n_{B}, U\right)\right] \hat{r} V=\frac{\partial \sigma_{S}}{\partial U}}
\end{array}\right.
$$

whose solutions lead to the following form of the matrix $\boldsymbol{Q}(x)$ (using Assumption 3):

$$
\boldsymbol{Q}\left(n_{A}, n_{B}, U\right)=\left(\begin{array}{lll}
\nu q_{12}-\frac{\partial \sigma_{S}}{\partial n_{A}} \frac{1}{\hat{r} V} & q_{12} & q_{13} \\
\nu q_{22}-\frac{\partial \sigma_{S}}{\partial n_{B}} \frac{1}{\hat{r} V} & q_{22} & q_{23} \\
\nu q_{32}-\frac{\partial \sigma_{S}}{\partial U} \frac{1}{\hat{r} V} & q_{32} & q_{33}
\end{array}\right)
$$

where the dependence with respect to $\left(n_{A}, n_{B}, U\right)$ of $\hat{r}$ and $q_{i j}$ has been omitted for the sake of simplicity of the notations. The form above of matrix $Q$ is obtained using (12) and (13). Indeed the general solution of the homogeneous system is given by:

$$
\boldsymbol{Q}_{h}\left(n_{A}, n_{B}, U\right)=\left(\begin{array}{ccc}
\nu q_{12} & q_{12} & q_{13} \\
\nu q_{22} & q_{22} & q_{23} \\
\nu q_{32} & q_{32} & q_{33}
\end{array}\right)
$$

and a particular solution is obtained by using (13):

$$
\boldsymbol{Q}_{p}\left(n_{A}, n_{B}, U\right)=\left(\begin{array}{cccc}
-\frac{\partial \sigma_{S}}{\partial n_{A}} \frac{1}{\hat{r} V} & 0 & 0 \\
-\frac{\partial \sigma_{S}}{\partial n_{B}} \frac{1}{\hat{r} V} & 0 & 0 \\
-\frac{\partial \sigma_{S}}{\partial U} \frac{1}{\hat{r} V} & 0 & 0
\end{array}\right)
$$

Let us now restrict ourselves to some of the solutions of (18) by setting

$$
q_{13}=q_{23}=q_{32}=0
$$

The remaining unknowns $q_{12}, q_{22}$ and $q_{33}$ shall be determined so as to meet the sign constraint (2). Since it is assumed that $\sigma_{S}$ is strictly decreasing along the system trajectories, (2) can be forced to be negative definite. Using the principal minors the sign constraint is hence equivalent to the following three inequalities:

$$
\begin{aligned}
0> & \left(\nu q_{12}-\frac{\partial \sigma_{S}}{\partial n_{A}} \frac{1}{\hat{r} V}\right) \\
0<4 & \left(\nu q_{12}-\frac{\partial \sigma_{S}}{\partial n_{A}} \frac{1}{\hat{r} V}\right) q_{22}-\left(q_{12}+\nu q_{22}-\frac{\partial \sigma_{S}}{\partial n_{B}} \frac{1}{\hat{r} V}\right)^{2} \\
0>q_{33} & {\left[4\left(\nu q_{12}-\frac{\partial \sigma_{S}}{\partial n_{A}} \frac{1}{\hat{r} V}\right) q_{22}-\left(q_{12}+\nu q_{22}-\frac{\partial \sigma_{S}}{\partial n_{B}} \frac{1}{\hat{r} V}\right)^{2}\right] } \\
& -q_{22}\left(\frac{\partial \sigma_{S}}{\partial U} \frac{1}{\hat{r} V}\right)^{2} .
\end{aligned}
$$

By using Assumption 2, it can be shown that a solution of the Brayton-Moser PDE (5) subject to the sign constraint (2) is given by the following expression for $\boldsymbol{Q}(x)$ :

$\boldsymbol{Q}\left(n_{A}, n_{B}, U\right)=\left(\begin{array}{ccc}\gamma & \frac{\partial \sigma_{S}}{\partial n_{B}} \frac{1}{\hat{r} V} & 0 \\ \frac{4 \alpha}{\nu} \gamma-\frac{\partial \sigma_{S}}{\partial n_{B}} \frac{1}{\hat{r} V} & \frac{4 \alpha}{\nu^{2}} \gamma & 0 \\ -\frac{\partial \sigma_{S}}{\partial U} \frac{1}{\hat{r} V} & 0 & \beta \frac{\left(\frac{\partial \sigma_{S}}{\partial U} \frac{1}{\hat{r} V}\right)^{2}}{4(1-\alpha) \gamma}\end{array}\right)$

where $0<\alpha<1$ and $\beta>1$ are scalar constants and $\gamma=\nu \frac{\partial \sigma_{S}}{\partial n_{B}} \frac{1}{\hat{r} V}-\frac{\partial \sigma_{S}}{\partial n_{A}} \frac{1}{\hat{r} V}$.

\section{CONCLUDING REMARKS}

A general methodology has been developed for solving the partial differential equation system that allows to write the system dynamics in the Brayton-Moser form. This methodology allows to find the set of solutions of the Brayton-Moser PDE system without taking into account the sign condition. In order to find a solution that satisfies the sign constraint, it is necessary to know a function that is decreasing along the system trajectories, which is far from being easy in any case. Nevertheless physical intuition can help, which leads to potential functions with physical meaning. It is also possible to solve the Brayton-Moser PDE system with the sign condition without choosing the a priori potential function candidate, as it has been done in [5]. The potential function results afterwards. However no general methodology has been found yet for doing this, and it becomes quite tedious when the dimension or the complexity of the nonlinearity increase. Future developments should investigate in this direction.

\section{ACKNOWLEDGMENTS}

This paper presents research results of the Belgian Network DYSCO (Dynamical Systems, Control, and Optimization), funded by the Interuniversity Attraction Poles Programme, initiated by the Belgian State, Science Policy Office. The scientific responsibility rests with its authors. Audrey Favache was a fellow student of the Belgian Fonds National de la Recherche Scientifique (FNRS). 


\section{REFERENCES}

[1] A.A. Alonso, B.E. Ydstie, and J.R. Banga. From irreversible thermodynamics to a robust control theory for distributed process systems. Journal of Process Control, 12:507-517, 2002.

[2] R.K. Brayton and J.K. Moser. A theory of nonlinear networks I \& II. Quaterly of Applied Mathematics, 22:1-33 \& 81-104, 1964.

[3] H.B. Callen. Thermodynamics and an Introduction to Thermostatics. John Wiley \& Sons, New York, $2^{\text {nd }}$ edition, 1985.

[4] V. Chellaboina, S. P. Bhat, W. M. Haddad, and D. S. Bernstein. Modeling and analysis of mass-action kinetics. IEEE Control Systems Magazine, 29:60-78, 2009.

[5] A. Favache. Thermodynamics and Process Control. $\mathrm{PhD}$ thesis, Université catholique de Louvain, Belgium, 2009.

[6] A. Favache and D.Dochain. Analysis and control of the exothermic continuous stirred tank reactor: the power-shaping approach. In Proc. $48^{\text {th }}$ IEEE Conference on Decision and Control, pages 1866-1871, Shanghai (China), 2009.

[7] A. Favache and D.Dochain. Power-shaping control of an exothermic continuous stirred tank reactor (CSTR). In Proc. International Symposium on Advanced Control of Chemical Processes (ADCHEM), pages 101-110, Koç (Turkey), 2009.

[8] Audrey Favache and Denis Dochain. Thermodynamics and chemical systems stability: The CSTR case study revisited. Journal of Process Control, 19(3):371-379, 2009.

[9] E. GarcÃa-Canseco, D. Jeltsema, R. Ortega, and J.M.A. Scherpen. Power-based control of physical systems. Automatica, 46(1):127-132, 2010.

[10] P. Glansdorff and I. Prigogine. On a general evolution criterion in macroscopic physics. Physica, 30:351-374, 1964.

[11] A. Isidori. Nonlinear Control Systems. Springer Verlag, London, $3^{\text {rd }}$ edition, 1995.

[12] D. Jeltsema, R. Ortega, and J.M.A. Scherpen. An energy-balancing perspective of interconnection and damping assignment control of nonlinear systems. Automatica, 40(9):1643-1646, 2004.

[13] D. Jeltsema and J.M.A. Scherpen. On mechanical mixed potential, content and co-content. In Proc. European Control Conference, pages 73-78, 2003.

[14] D. Jeltsema and J.M.A. Scherpen. A power-based description of standard mechanical systems. Systems \& Control Letters, 56(5):349356, 2007.

[15] B.M. Maschke, R. Ortega, and A.J. van der Schaft. Energy-based Lyapunov functions for forced Hamiltonian systems with dissipation. IEEE Transactions on Automatic Control, 45(8):1498-1502, 2000

[16] B. O'Neill. Elementary Differential Geometry. Academic Press, New York, 1997.

[17] R. Ortega, D. Jeltsema, and J.M.A. Scherpen. Power shaping: A new paradigm for stabilization of nonlinear RLC-circuits. IEEE Transactions on Automatic Control, 48(10):1762-1767, 2003.

[18] R. Ortega, A.J. van der Schaft, I. Mareels, and B. Maschke. Putting energy back in control. IEEE Control Systems Magazine, 21(2):18-33, 2001.

[19] R. Ortega, A.J. van der Schaft, B.M. Maschke, and G.Escobar. Interconnection and damping assignment: passivity-based control of portcontrolled Hamiltonian systems. Automatica, 38(4):585-596, 2002.

[20] I. Otero-Muras, G. Szederkényi, A.A. Alonso, and K.M. Hangos. Dynamic analysis and control of chemical and biochemical reaction networks. In Proc. International Symposium on Advanced Control of Chemical Processes, pages 165-170, Gramado (Brazil), April 2006.

[21] R. Perry and D. Green. Perry's Chemical Engineers' Handbook. McGraw-Hill, New York, $7^{\text {th }}$ edition, 1997. 